\title{
Proficient Handling and Restraint of the Laboratory Animal Rat (Rattus Norvegicus) Facilitate Essential Biochemical and Molecular Level Studies in Biomedical Sciences
}

\author{
${ }^{1}$ Mudassar Manzoor, ${ }^{2}$ Sana Raza, ${ }^{3}$ BushraChaudhry \\ 1, 2,3 (Biological and Biomedical Sciences, Aga Khan University Medical College, Pakistan)
}

\begin{abstract}
The laboratory rat is an important animal model which has been used extensively in the fields of biological, pharmaceutical, behavioral and biomedical sciences. There are several laboratory procedures which are implemented on this model repetitively. These procedures require proper handling and restraint of the rat. A good amount of general information is available at several places on web. Knowledge about safe and effective rat handling techniques and methods are mandatory to learn before starting experiments on animal models. Avoidance of stress and discomfort of the rat is very important for the overall outcome of an experimental study. Here we address and review someessential techniques to handle difficulties of working with the laboratory rat (RattusNorvegicus) using our first-hand experience from an animal care and safety perspective in moderately available animal facility especially for developing countries.
\end{abstract}

Keywords:Handling and Restraint techniques,Rats,Anesthesia, Euthanasia, Safety

\section{Introduction}

A laboratory animal model is a living animal in which a biological or behavioral phenomenon can be studied by investigating the pathological and biochemical processes which are similar to phenomenon in humans or any other animal species of interest [1]. Animal models are also used for determining the various biochemical pathways and behavioral approaches in the field of biomedical research. There are a number of animal models including mammalian (rodents, rabbits, pigs, cats, dogs, chimpanzee) and non-mammalian (zebra fish, fission yeast, filamentous fungus, social amoeba, round worm, water flea, fruit fly, frog, chicken) which are used for performing different experimental procedures.

The laboratory rat (Rattus Norvegicus) belongs to order Rodentia and is a commonly used animal model. The breeding and housing of this rat is carried out for medical and scientific research purpose. It is believed that the laboratory rat was the first animal which was domesticated only for the purpose of scientific research [2]. The rat which is used for biomedical research all over the world is considered to be a sharp, swift and intelligent laboratory animal as compared to other laboratory rodents likes mouse, gerbil and hamster. Its behavior can be judged easily when it is handled and restraint appropriately. Some of its characteristics such as cost effectiveness, easy transportation, high fertility rate, shorter life span and to be housed in a limited space make it worth to use. Because of its various unique characteristics, it is preferred to use for performing various research based studies especially in the field of medical sciences [3]. There are different outbred and inbred strains of rats which are being adapted to use in laboratories. Common outbred strains are Wistar rat and Sprague-Dawley rat. Inbred strains are Brown Norway, Lewis, Dark Agouti, Athymic Nude, Buffalo and Fisher 344 rats. Due to some outstanding potential, usually outbred strains are more used as compare to inbred strains [4].

Rats usually show irritation and bad temper on being treated by inexperienced handlers and they can suffer from severe stress. Outbred strains of rat, either male or female, are normally quiet, docile and easy to handle. It is suggested to familiarize with these rats at their early life stages and handled them regularly if possible. On the other hand, some inbred strains can prove themselves grumpier and they will need extra care and attention. It is not easy to handle and restrain them [3]. It is necessary to handle and restraint the rat in a particular position for performing different experimental trials. Therefore, it is necessary to adopt such techniques that can be pleasant and harmless for the under-trial rats. Safety of the rats must be ensured by reducing any possibility of physical injury and stress to the rat. It can be achieved through efficient and appropriate rat handling. Due to incorrect handling, they can easily come into stress and can react desperately by biting or scratching handler's body. The applied force and technique must be proper and suitable for the animal to be restrained [5]. Here, we will review some rat handling techniques and methods which can be proved very helpful particularly for the safety of rat and its handler. The rat identification, sex determination, transport, handling, restraint, substance administration, blood collection, anesthesia and euthanasia will be discussed. 


\section{Methods}

Methods by which rats can be identified easily in an animal facility can be selected from following techniques.

\subsection{Sex determination}

Sex determination of the rats is carried out by comparing the ano-genital distance between the genital papillae and the anus which is greater in males than in females [6]. Fig.1 $\mathrm{a}$ and $\mathrm{b}$

\subsection{Cage cards}

Individual rats, a single breeding pair or groups of the same rat strain can be recognized by placing cards on their cages containing the detailed information about strain, species, age, selected for experiment date etc. Fig. 2a

\subsection{Temporary marking}

This method is applied for short term individual rat identification by writing numbers, bars or other distinguishable marking on the tails or on eras of the rats within a cage using non-toxic, permanent marker. If temporary marking is to be used for more than a week then marking should be repeated at least twice a week. Fig. 2b

\subsection{Ear punching}

Restrain the rat carefully and place ear punch in desired location. Firmly and quickly punch a hole in the ear to avoid an incomplete cut. Ear punching cannot be applied to the rats under age of two weeks. Fig. $3 \mathrm{a}$

\subsection{Ear tag}

Tag is placed low on the pinna so that it rests against the rat. As a result it does not bend the ear, catch on the cage or cause the rat to hold its head in an uneven way. Tags should be of appropriate size and rinsed with $70 \%$ ethanol before use. Fig. $3 \mathrm{~b}$

\subsection{Tattooing}

Local or general anesthesia is suggested for carrying out this method as an electric tattoo machine is used to write numbers on the tail. It must be ensured that the needles and other equipment are sterilized for performing tattooing method. Fig. 3c

\subsection{Micro-tattooing}

In this technique, tattoo ink is injected in the toe pads or the ears by using a micro-tattooer. A simple code must be used to limit the number of toes tattooed.

\subsection{Microchips}

Rats can also be identified by implanting the microchips by using the implanter which injects the microchip subcutaneously in the neck area between shoulder blades. General anesthesia and analgesia must be given to implant microchip in animals more than 3 weeks of age. Microchips can be reused after proper cleaning and sterilization. Several companies provide theses microchips including AVID, NONATEC, Kent Scientific Corporation and Bio Medic Data Systems, Inc. Fig. 3d

\section{Handling and Restraint Techniques}

To deal with an animal in a direct or indirect way and with or without touching the animal is termed as Handling. When an animal is immobilized by keeping itself or its body parts, in a relaxed and secure position by using hand or physical device is known as Restraint [7]. In an extensive research study, there are many experimental conditions where it is necessary to restrain the rats by hand. Restraint is usually mandatory for making observations, substances administration, blood collection or rat transportation. For safe and effective handling, the handler must have comprehensive knowledge of animal's physiology; anatomy and responsive behavior [8]. There are some techniques and methods which are routinely being used in laboratories at global level.

\subsection{Rat transport restraint}

Rat is transported out of its cage by gently gripping the base or center of its tail with the thumb and index finger and is placed in a new cage, weight balance or on a rough surface. Serious injury can result to the rat if picked up by the tip of the tail since the tail skin may pull off. It is advised that not to suspend the rat by the tail or the upper body for a long time period [2].The activities like transporting and trapping create high 
stress levels in rats. It is suggested that to give them some time to calm down before any other additional restraint [4]. Fig. 4a

\subsection{Shoulder or Two fingers restraint}

In this restraint technique, the laboratory rat is being approached dorsally and its thorax is surrounded just behind front legs by using thumb and forefinger firmly. Thorax of the rat can be supported with remaining fingers and it must be observed that rat is breathing normally under firm grip [9]. Fig. 4b

\subsection{Encircling or Three fingers restraint}

This technique is used to grasp the rat around the thorax with thumb and index finger. Thumb will slide across the ventral thorax and underneath one of the forelegs. Its forelimbs are extended by pushing the elbows to cross the two front paws. Fig. $4 \mathrm{c}$

\subsection{Grip or Four fingers restraint}

The index and middle finger of the handler slide together along both sides of rat's head to the extent that its head and jaw bones are seized between the knuckles of both fingers. Then thorax is grasped by thumb and remaining fingers under both fore limbs of rat. In case of more body weight, the lower body can also be supported with other hand. Fig. 4d

\subsection{Neck hold or Scruff restraint}

The tail of rat is grabbed with the thumb and index finger of non-dominant hand to keep the rat in a fixed position and palm of the other dominant hand is placed on the rat's back. Loose skin along the dorsum including that along the dorsal neck is being gathered by using thumb and fingers. The thumb and index finger are employed to stabilize the head and the remaining three fingers are used to stabilize the trunk. This technique is considered to be the least used despite of its effectiveness. Fig. 5a

\subsection{Restraint tubes or Boxes}

This technique was designed for easy access to the tail. Rats usually enter these tubes happily.

The rat is grasped gently around the thorax with the thumb and fingers under each of the front legs. The rat's head is placed into the opening of the restraint tube. Rat is released and grip is maintained on its tail. For providing required restraint, securing block is fixed in proper slot. Fig. $5 \mathrm{~b}$

\subsection{Cone restraint} first. Fig. 5c

Grab the base of the tail firmly with the thumb and index finger and place the rat in cone with the head

\subsection{Towel or Cloth restraint}

The rats that are very nervous, rude or aggressive can be restrained by using restraint devices like towels, cotton stockinet, and gloves. This method has the advantage of controlling the hind limbs which prevents potential scratching. Fig. $5 \mathrm{~d}$

\subsection{Oral gavages}

\section{IV.Substance Administration and Injection Techniques}

It is possible to administer different substances orally by specially designed gavage tubes which are made up of stainless steel and have ball tips. The length of the gavage tube must be according to the distance which is measured from the tip of nose to the last rib [2]. Rat is restrained properly and tube is inserted into the mouth to induce swallowing. The tube should pass easily down the esophagus and with no resistance. If there is any resistance then it indicates the insertion of tube into trachea which can cause sudden death. In such case withdraw the tube and try again. But there should be no more than two attempts for avoiding any stress. Maximum amount which can be administrated orally is $20 \mathrm{ml} / \mathrm{kg}$ [10]. Fig. 6

\subsection{Injections}

It is recommended to use disposable sterile syringes and needles for injecting the substances. Moreover, the smallest gauge needle must be selected for reducing the tissue trauma and injection discomfort to rats for all the injecting procedures. Some of the injecting techniques are mentioned here:

\subsubsection{Subcutaneous injection}

For subcutaneous injection, such sites are preferred where skin is loose and mobile especially along the scruff or flank. The skin between the shoulder blades is lifted in such a way that a tent is formed and needle is 
inserted in its base. The angle of the needle must be one dimensional and low so that the underlying tissues and blood vessels can be confined from any damage. Aspirate the needle to check the right position of needle inside the tent and if there is no blood in needle then inject the substance. The amount of drug up to $5 \mathrm{ml} / \mathrm{kg}$ can be injected by this technique [9]. Fig. 7a

\subsubsection{Intramuscular injection}

Intramuscular injection in the laboratory rat is not preferred as big muscles are lacking and this technique cannot perform easily. It is suggested that to use very small amounts of drug through this rout. Despite of the weight of rat, total amount should not be more than $0.1 \mathrm{ml}$ [10]. Insert the needle in the muscles running along the back of the rat's leg and aspirate it for confirming the right insertion. If blood does not come out after aspiration, then inject the substance. Fig. $7 \mathrm{~b}$

\subsubsection{Intravenous injection}

It is regarded as one of the most commonly used injection technique. Rat is restrained in a Plexiglas restrainer and its tail is warmed by exposing it briefly to a heat lamp or immersion in warm water for dilating the target veins. Animal should be closely observed to avoid hyperthermia. After dilation, the lateral vein can easily be located and needle entry is possible. The needle is penetrated and aspirated to check the presence of blood before injecting. Bleeding should be stopped before returning the rat into cage. The amount of drug up to 5 $\mathrm{ml} / \mathrm{kg}$ can be injected by this technique [10]. Fig. 7c

\subsubsection{Intraperitoneal injection}

Intraperitoneal injection is also another safe and effective technique commonly used in laboratories. Short needles are recommended so that any damage to abdominal organs can be avoided. Posterior quadrants of the abdomen are being selected for needle insertion. The syringe must be aspirated to avoid any penetration in the gut or bladder. When there is no fluid in the needle then inject the substance. The amount of drug up to 5 $\mathrm{ml} / \mathrm{kg}$ can be injected by this technique [10]. Fig. $7 \mathrm{~d}$

\subsubsection{Intradermal injection}

Anesthesia must be given to rat prior intradermal injection. Hair on the back are clipped and injection site is prepared with alcohol swab. The needle is inserted at an angle of $30^{\circ}$ between the layers of skin of the back. The syringe is aspirate to insure proper placement. Any sign of blood or any other fluid in the needle shows improper placement. Maximum volume of $100 \mathrm{ul} / \mathrm{site}$ must be administrated to avoid any damage to tissues [9].

\section{V.Blood Collection Methods}

Blood can be collected from different locations of a rat's body depending upon the desired volume of blood. Whatever the method is going to be used, it must be assured that it is according to IACUC recommendations. Sterile syringes and needles must always use for all the procedures.

Serial or repeated sample collection is carried out from saphenous vein (hind leg) and tail vessels and terminal blood collection is carried out by cardiac puncture [11].The average circulating blood volume in rat is $70 \mathrm{ml} / \mathrm{kg}$ and $10 \%$ of its volume can be collected within a 3 to 4 weeks period. For repeated sample collection, $1 \%$ of total volume can be withdrawn every 24 hours [9].

\subsection{Lateral saphenous vein}

It is relatively quick method to collect blood from lateral saphenous vein [12]. Rat must be anesthetized prior to blood collection. A lubricant ointment like petroleum jelly or ophthalmic ointment must be applied to lateral part of tarsus to smooth the hair. Grasp the skin of thigh firmly to extend the leg and raise the vessel. Puncture this vessel with a $25 \mathrm{~g}$ needle and collect blood in a small tube or a syringe. Light pressure is applied to the puncture site to stop bleeding about 1-2 minutes and decrease hematoma. This technique is used for repeated sample collection and average blood collected ranges between 0.5 to $1 \mathrm{ml}$.

\subsection{Lateral tail vein}

Blood sampling from tail vein is also another quick and simple method [13]. It is relatively nontraumatic and can be done without anesthesia but effective physical restraint is required. Tail of the rat is warmed before collecting the sample. When vein is clearly visible then insert the needle and draw the blood. Apply light pressure to site for approximately 1-2 minutes to stop bleeding and decrease hematoma. Nearly $1 \mathrm{ml}$ blood can be collected from tail at one time. This method can be applied to ventral or dorsal Artery. 


\subsection{Ventral tail artery}

This method also requires anesthesia for blood sample collection from ventral tail artery. The rat is placed in dorsal position for the procedure and the ventral puncture site is prepared aseptically. Nearly one third of the distance from the tail base is recommended for puncture site and needle is inserted at a $30^{\circ}$ angle on the ventral midline of the tail [14]. A "pop" should be felt on entering the needle in artery. As blood drips from the syringe, it can be collected in a microtainer tube [15].

\subsection{Blood Collection by Retro-orbital bleed}

Rat needs to be anesthetized before applying this technique and then local anesthesia is also given to target eye by using anesthetics like tetracaine or proparacaine. It takes nearly 30 second for these anesthetics to become effective and anesthesia lasts for about 5-10 minutes. Hematocrit tube is placed at the medial canthus of the eye. After insertion behind the eye, rotate this tube on its axis on the back of the orbital until a pop is felt and blood flows into tube. Sterile eye ointment is filled after blood withdrawal. Although this is a fine procedure but it is not commonly used now[2].

\subsection{Tail tip snip}

This method can be used for repeated sample collection. Sharp iris scissor is used to cut the distal end of tail. Bloodis collected into a collecting tube and pressure is applied to stop the bleeding. If it is desired to repeat the sample collection then outer layer of tail can be detached to resume bleeding.

\subsection{Intracardiac (IC) puncture}

Intracardiac (IC) Puncture method is performed for terminal sample collection and is followed by euthanasia. In this method, the needle is inserted just behind the forelimb because the heartbeat is strongly felt there. The heart is located nearly under the elbow when leg is placed on a normal anatomic position. The needle must be advanced firmly and aspirate the syringe slowly. Generally expected blood volume is $3 \%$ of body weight. Prior anesthesia is mandatory to decrease the pain.

\section{VI.Methods of Anesthesia}

There are basically two methods of anesthetic delivery to rats which are parenteral and inhalation.

\subsection{Parenteral anesthesia}

In this method, intraperitoneal, intramuscular and intravenous routes of administration are being used for injecting anesthetics. This method is rapid and cost efficient but recovery is less.

\subsection{Inhalation anesthesia}

The volatile anesthetic agents are delivered to the rat through the respiratory tract. In this method, the depth and duration of anesthesia can easily be controlled which results into greater survivability. Moreover, such anesthetic agents can be administered which cause less metabolism biotransformation and thus help to avoid the upsets in the study results.

\section{Methods of Euthanasia}

Euthanasia means a gentle or peaceful death and it is performed by humane killing of animals with less pain, fear and distress. At the end of an experiment, rats are normally killed in the laboratories for obtaining blood and other tissues for research purpose [16].

According to the 2007 Report of the American Veterinary Medical Association Panel on Euthanasia [17], the methods of euthanasia are classified as acceptable, conditionally acceptable and unacceptable methods. Acceptable methods are those that are merely based on producing a humane death like cervical dislocation, carbon dioxide $\left(\mathrm{CO}_{2}\right)$, barbiturate and inhalant anesthetic overdose. The methods which do not always produce humane death due to the safety concerns, types of technique or may be more likely for the handler are classified as conditionally acceptable methods. Unacceptable methods are those methods which always produce brutal death and may also be a potential risk for handler. Some of the acceptable and conditionally acceptable methods are discussed here.

\subsection{Cervical dislocation}

Place a closed scissor like object firmly across the rat's neck at the base of the skull and quickly perform cervical dislocation by grasping the tail near the base and sharply pulling away from the body. The rat must be dead immediately. This procedure is only for use in those rats whose body weight is less than 200 grams. This method is acceptable only when rats are anesthetized and must be performed by a skilled handler. 


\subsection{Carbon dioxide $\left(\mathrm{CO}_{2}\right)$ overdose}

Euthanasia chamber should be slowly filled with compressed $\mathrm{CO}_{2}$ gas but not pre-filled. The number of rats should not be exceeded in the chamber. The rats should quickly defer to death on placement in the chamber. If rats show any movement then increase the $\mathrm{CO}_{2}$ amount gradually. The chamber must be disinfected after use. This method can be applied on all rats except neonates.

\subsection{Barbiturate overdose}

Injectable euthanasia solution or sodium pentobarbital $(100 \mathrm{mg} / \mathrm{kg})$ can be used to euthanize all the rats by giving them overdose. These preparations can be administered intraperitoneally or intravenously. These compounds do not administer subcutaneously or intramuscularly.

\subsection{Inhalant anesthetic overdose}

This method is recommended for neonates but must be followed by an adjunctive method. The rats are placed in a closed chamber containing cotton or gauze soaked with an appropriate amount of inhalation anesthetic which can be halothane, enflurane, isoflurane, sevoflurane, desflurane. The respiration is ceased due to vapors inhalation and ultimately death occurs. Inhalant anesthetic exposure to humans can be hazardous. A fume hood must be used to perform this method.

\subsection{Decapitation}

This is a conditionally acceptable method which is very useful for obtaining the undamaged and uncontaminated brain, kidney and pancreatic tissues from rats [17]. Decapitation without prior anesthesia must be scientifically justified. Plastic restraint cones can be used for minimizing the stress of rats. This method requires the use of specially designed equipment with sharp blades called guillotine.It is recommended that the animal handlers must properly be trained and skilled in using all these euthanasia methods.

\section{Waste Disposal}

An animal house facility usually produces a large amount of waste and carcasses which must be disposed of regularly. Accessibility of waste boxes and containers with tight lids must be assured at animal research facility. The entrails and dead bodies of laboratory animals must be discarded properly for avoiding the odor or groundwater and waterway contamination which can cause health problems.

Waste, carcasses, tissues and organs of animals should be disposed of not more than 24 hours after confirmation of death. If immediate waste disposal is not possible then it should be stored in a separate, insects-free and disinfected waste storage area. This storage area should be separated from the animal holding and clean equipment areas.

Non-bio hazardous waste material must be removed by incineration and land-fill procedures which should be carried out according to the guidelines of relevant institutional biosafety standards and legislation. Hazardous waste which includes carcasses and tissues of animals exposed to radioactive, bio-hazardous, toxic and microbiological agents, must be sterilized appropriately prior to removal by incineration or land-fill procedures [18].

\section{Safety Practices}

There are many risks and hazards which are associated with the rat handling procedures. The rat's handlers must be aware of these risks which can be harmful for their health. With proper handling and appropriate knowledge of rat's behavior, these factors can be reduced to greater extend. The primary risk associated with rat handling is of bites and scratches which can offer serious injuries to the handlers. Such injuries can easily be avoidable by proper rat handling techniques and methods. The rat can suffer from pain, discomfort and distress because of untrained handling. This can aggravate an irritable response which can produce undesirable experimental variables. It can also make possible for the rat to cause injury upon its poor handler. Therefore, it is suggested that to approach the animal calmly and quietly [19] and the handler must be trained enough about the animal handling techniques.

An estimated 37\% of individuals who work with laboratory animals may eventually develop an allergy to laboratory animals [20], which is manifested by reddened, itchy eyes, nasal symptoms and skin rashes. Asthma can also be developed in the persons who are already suffering from animal-associated allergy and some cases of Anaphylaxis are also reported [21]. It is suggested that the handler must wear the facemask, gloves, shoe coverings and lab coats before entering animal house. It cannot only provide protection from the allergens but also from zoonotic diseases. 


\section{Conclusion}

Knowledge disseminated about handling and restraining of the rat is important for the animal welfare and for beginners to perform various experimental studies.This information isalso very helpful in procedures like anesthesia, blood collection, substance administration and finally euthanasia. It must be ensured that the rat must be suffered from less stress, pain and discomfort. This purpose can be achieved by learning sufficient knowledge to handle animals and must have training and well awareness of animal behavior and response to assess the effects during experimental studies to avoid false positive observations and results.

\section{Acknowledgements}

We thank you Dr. Samara Bashir for critical reading and useful suggestions of the manuscript. We also thank you for financial funding from Higher Education Commission, Pakistan, through grant NO. 20$1713 / \mathrm{R} \& \mathrm{D} / \mathrm{HEC} / 2010$. Thanks are also extended to the staff of Animal House, responsible for the wellbeing of the animals, used in this study. SR, participated as a research volunteer scholar of Quaid-e-Azam University, Islamabad, Pakistan BC is the author of correspondence.

\section{References}

[1] S.Wessler, Introduction: What is a model? Committee on Animal Models for Thrombosis and Hemorrhagic Diseases; Institute of Laboratory Animal Resources. Animal models of thrombosis and hemorrhagic disease. National Institutes of Health, Bethesda, MD, 1976, 11-16.

[2] M.I. Perret-Gentil, Rat Bio methodology. Hands-on rat bio methodology workshop in the laboratory animal resources center (LARC).The University of Texas, San Antonio, TX, 2005.

[3] G. J. Krinke.The Laboratory Rat (Handbook of Experimental Animals), in G.R. Bullock and T.E. Bunton, (Ed.) (New York: Academic Press, 2000)

[4] P.K.H.Chow, R.T.H. Ng and B.E Ogden, Using Animal Models in Biomedical Research: A Primer for the Investigator (Singapore: World Scientific Publishing Co.Pte.Ltd,2008)

[5] M. E.Fowler,Restraint and handling of wild and domestic animals (Iowa State University Press, Iowa, IA, 1978)

[6] K. A.Murray, C.Pekow and G. L Borkowski, Laboratory Animals: Rodent Anesthesia \& Analgesia. Laboratory Animal Medicine and Science - Series II. Health Sciences Center for Educational Resources, University of Washington, Washington, WA, 2000.

[7] T.Buerge and T. Weiss, Handling and Restraint. In: Hans J Hedrich.Gillian Bullock. The Laboratory Mouse(Amsterdam; Boston: Elsevier Academic Press, 2004)

[8] M. E. Fowler,Zoo and wild animal medicine (Philadelphia: W.B. Saunders Company,1978)

[9] M. S. Rand, Handling, restraint, and techniques of Laboratory rodents. Department of Animal Care, University of Arizona, Tucson, AZ, 2010 .

[10] H. B. Waynforth, P.Brain, T.Sharpe, D. F.Stewart, K. A. Applebee andP. G. G. Darke,Good Practice Guidelines: Administration of Substances (Rat, Mouse, Guinea pig, Rabbit).Series 1 (1). (Laboratory Animal Science Association, Tamworth, Staffordshire, 1998)

[11] Anonymous, Animal Use Training Session: Rat Lab Handout. Animal Use Training Program, Department of Comparative Medicine, University of Washington, WA, 2010.

[12] A.Hem, A.J.SmithandP. Solberg, Saphenous vein puncture for blood sampling of the mouse, rat, hamster, gerbil, guineapig, ferret and mink. Lab Animal, 32, 1998, 364-368.

[13] J.Bazare, M.L. Leamons and J.F. Young, Sampling methods for pharmacokinetic studies in themouse. J Pharmacol Methods, 5, 1981, 99-120.

[14] C.Bihunm and L. Bauck, Basic anatomy, physiology, and clinical techniques, in K.E. Queensberry and J.W. Carpenter (Ed.), Ferrets, Rabbits and Rodents: Clinical Medicine and Surgery. (St Louis, MO: Saunders, 2003) 286-298.

[15] J.O. Joslin,Blood Collection Techniques in Exotic Small Mammals. Journal of Exotic Pet Medicine. 18, 2009, 117-139.

[16] B Close, K. Banister, V. Baumans, E.M. Bernoth, N.Bromage, J. Bunyan, W. Erhardt, P. Flecknell, N. Gregory, H. Hackbarth, D. Morton and C. Warwick, Recommendations for euthanasia of experimental animals: Part 1.LaboratoryAnimals, 30, 1996, 293-316.

[17] American Veterinary Medical Association (AVMA), The 2007 American Veterinary Medical Association Guide lines on Euthanasia, 2007, available at:www.nd.edu/ ndflsc/SOP Downloads.html

[18] University Animal Care Committee, Module 1-The Laboratory Rat. Laboratory Animal Bio-methodology Workshop. McGill University, Montreal, Canada, 2009.

[19] T Sharp, G. Saunders and B. Mitchell, RES002 Restraint and handling of pest animals used in research. (New South Wales Department of Primary Industries, 2007). Available electronically at: http://www.environment.gov.au/ biodiversity/ invasive/ publications/ threatabatement projects/pubs/46217-operating-procedure-2.pdf

[20] G.E. Davies, A.V. Thompson, Z. Niewola, G.E. Burrows, E.L. Teasdale, D.J. Bird andD.A. Phillips, Allergy to laboratory animals: a retrospective and a prospective study. British Journalof Industrial Medicine, 40, 1983, 442-449.

[21] E.L. Teasdale, E.G. Davies and R. Slovak, Anaphylaxis after bites by rodents.British Medical Journal, $286,1993,1480$. 

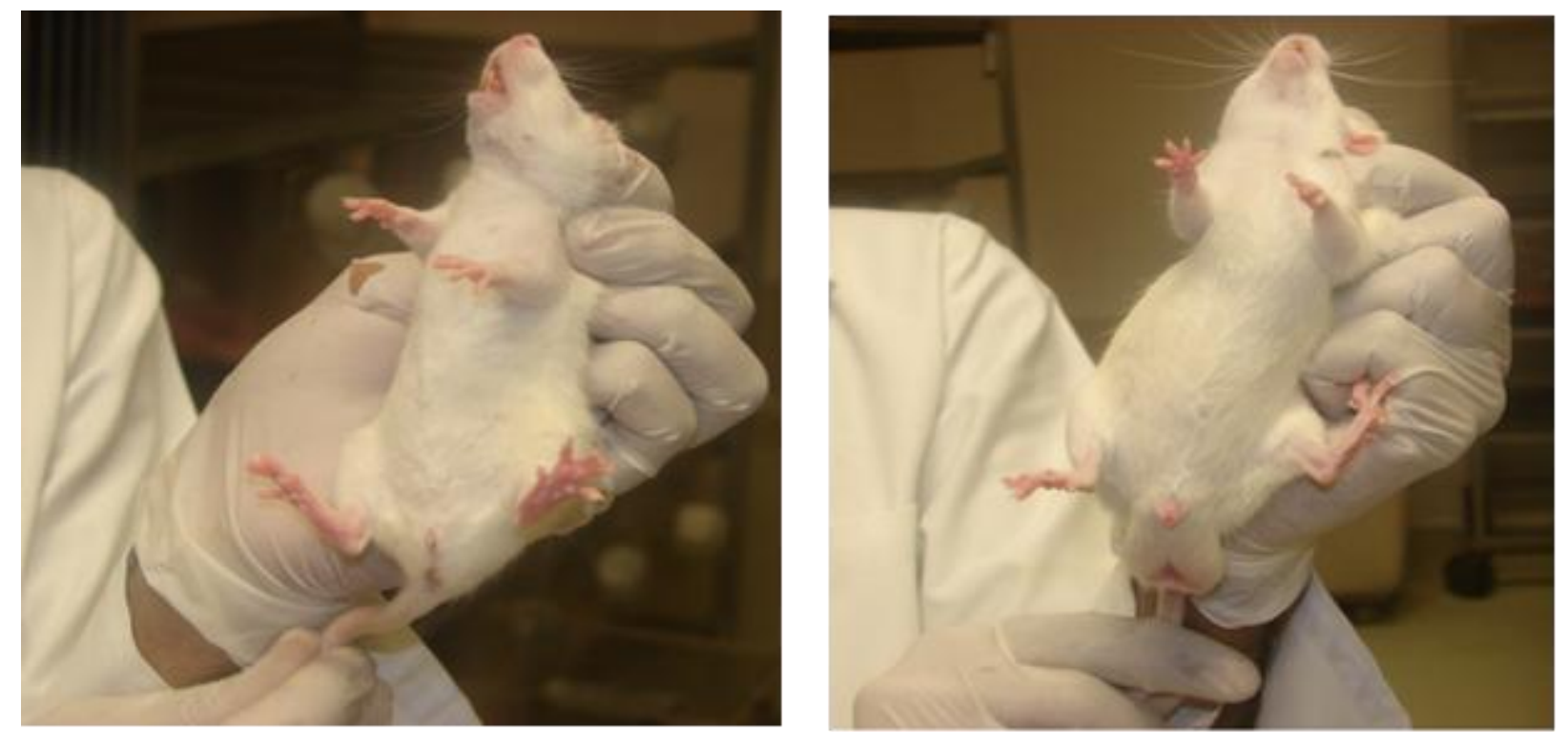

Fig.l: Sex determination method, a: female rat genitalia, b: male rat genitalia indicating the difference of the distance. (Pictures generated at Aga Khan University, Karachi, Pakistan)
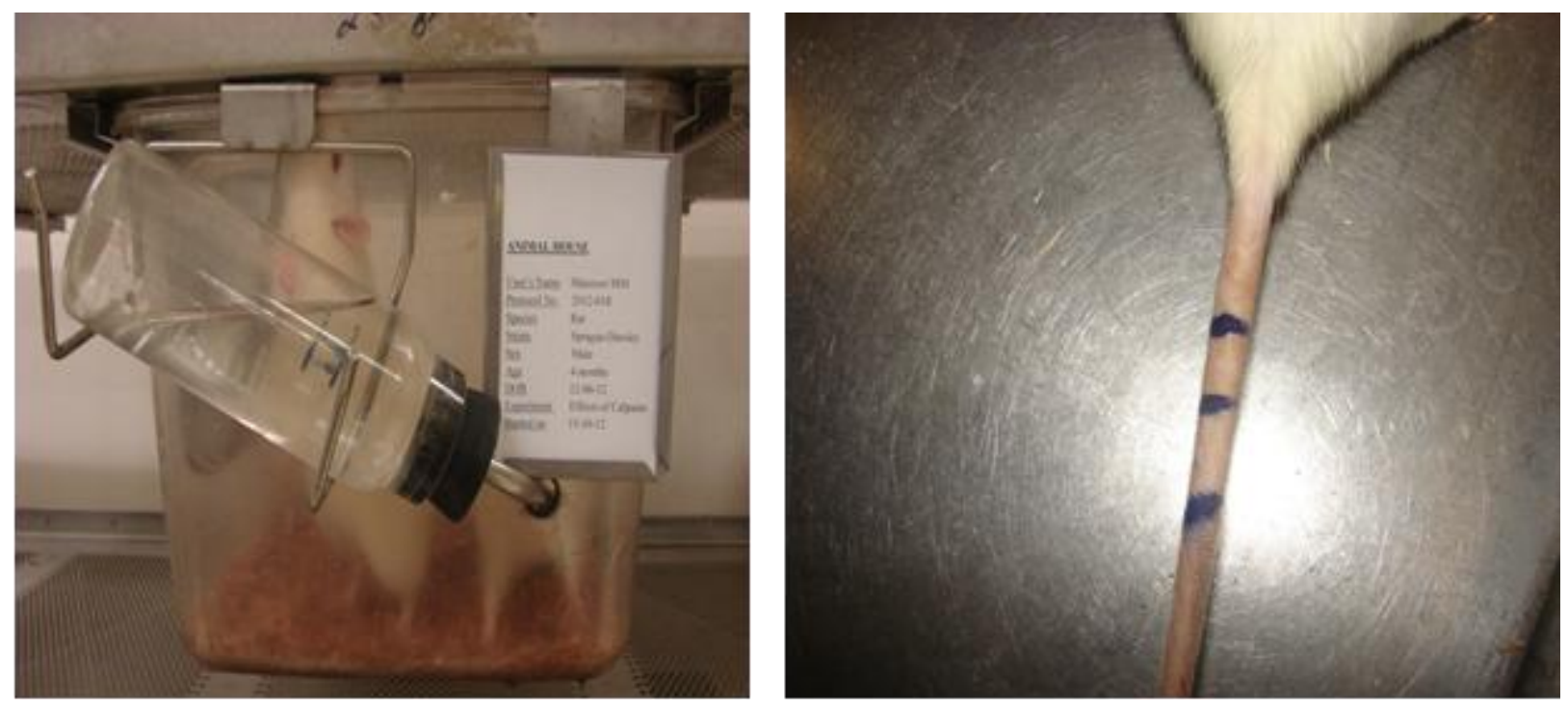

Fig.2: Rat identification method a: cage card comprising the detailed information, $b$ : tally marks on the rat's tail representing the temporary marking. (Pictures generated at Aga Khan University, Karachi, Pakistan) 

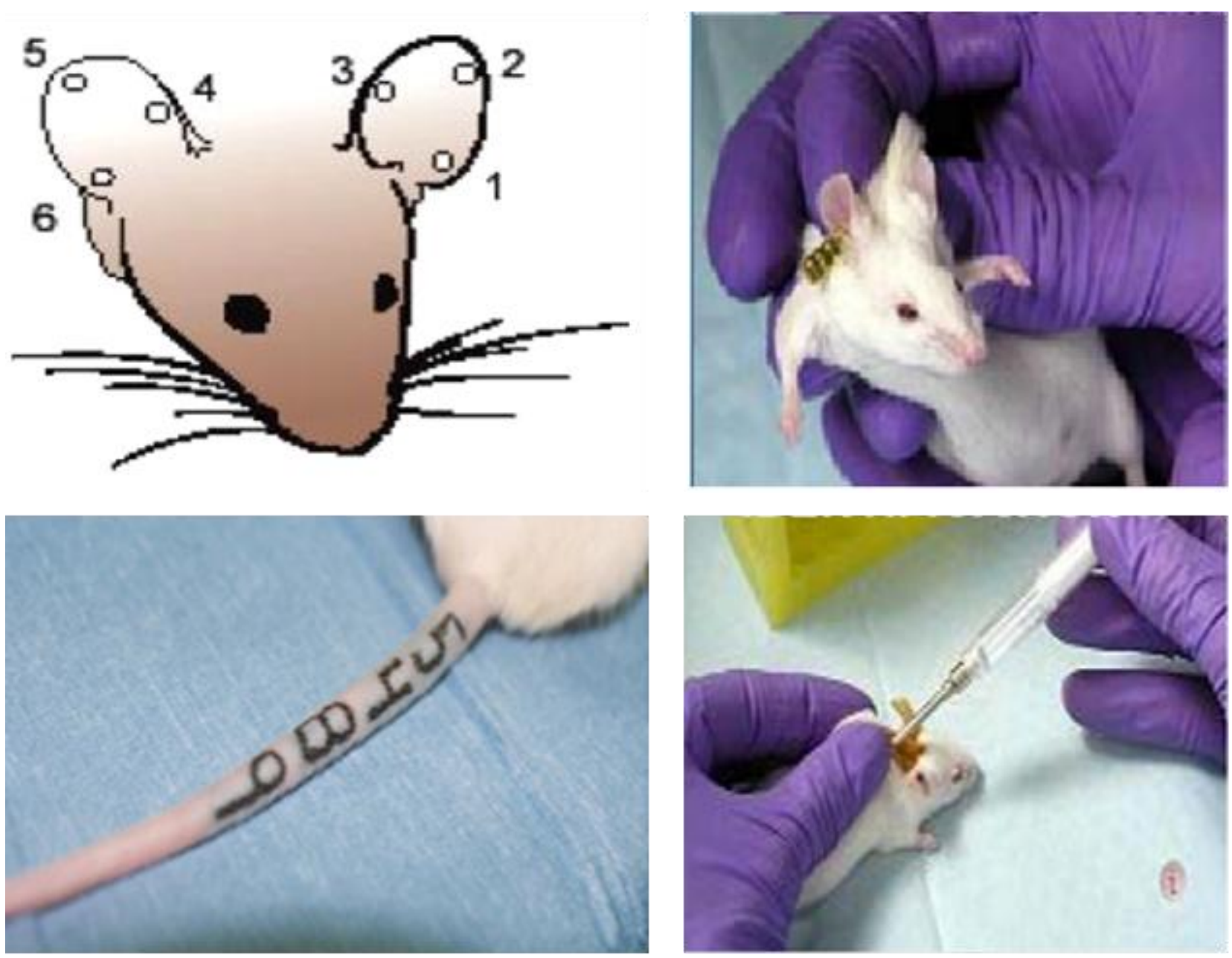

Fig3: Rat identification method a: specific holes in the ear demonstrating ear punch method, b: ear tags are being placed at the pinna c: Numbers are tattooed on the tail for tattooing purpose, d: microchips implantation method is highly effective but not cost efficient (adapted from http://images.banevillaflor.multiply.multiplycontent.com ). 

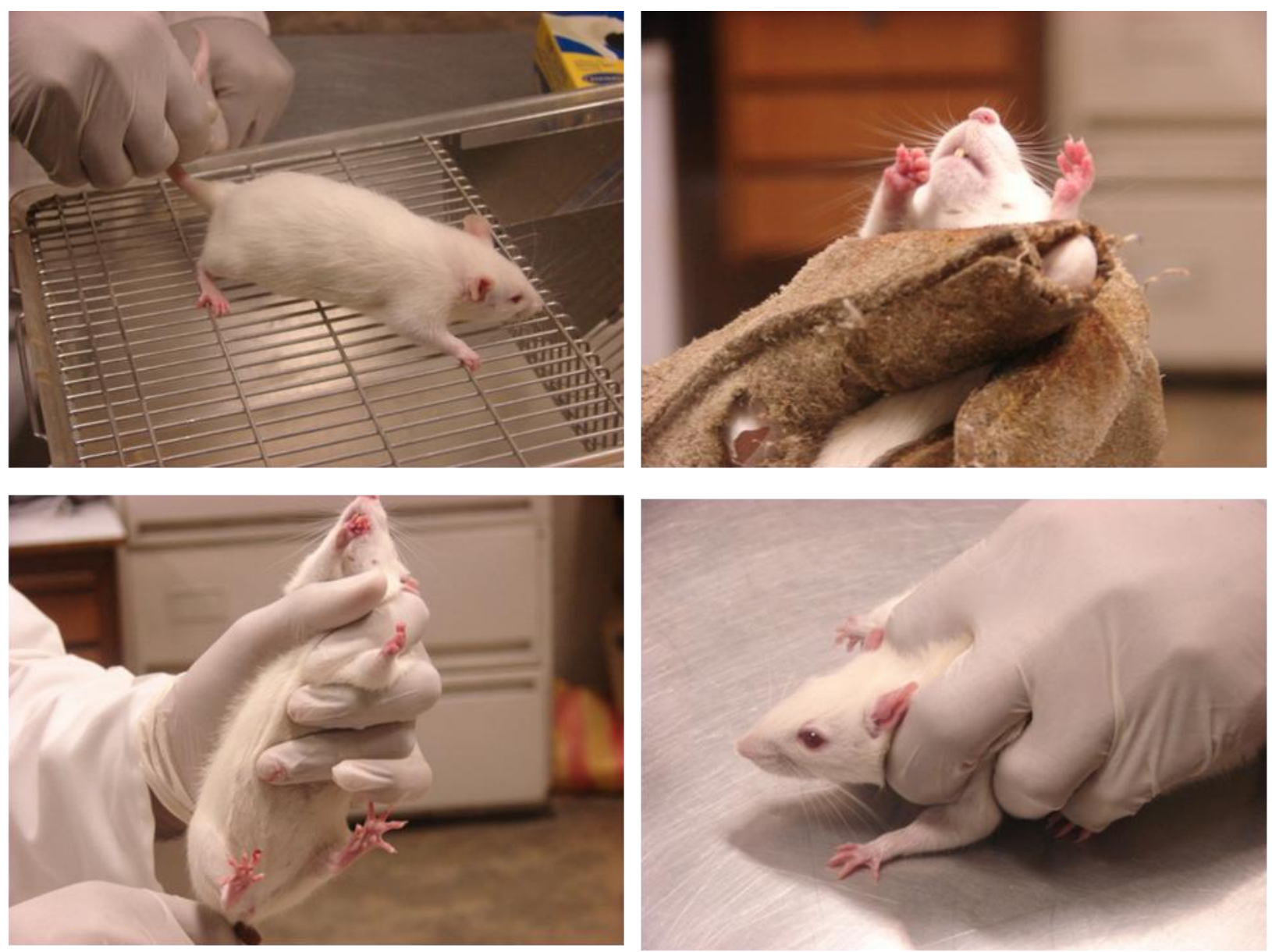

Fig.4: Handling and restraint techniques, a:rat's tail must be gripped at the base or center for transport restraint, b: shoulder restraint which is very much effective for substance administration, c: showing a commonly used encircling restraint, $d$ : Rat's head and jaw is grasped by grip restraint. (Pictures generated at Aga Khan University, Karachi, Pakistan) 

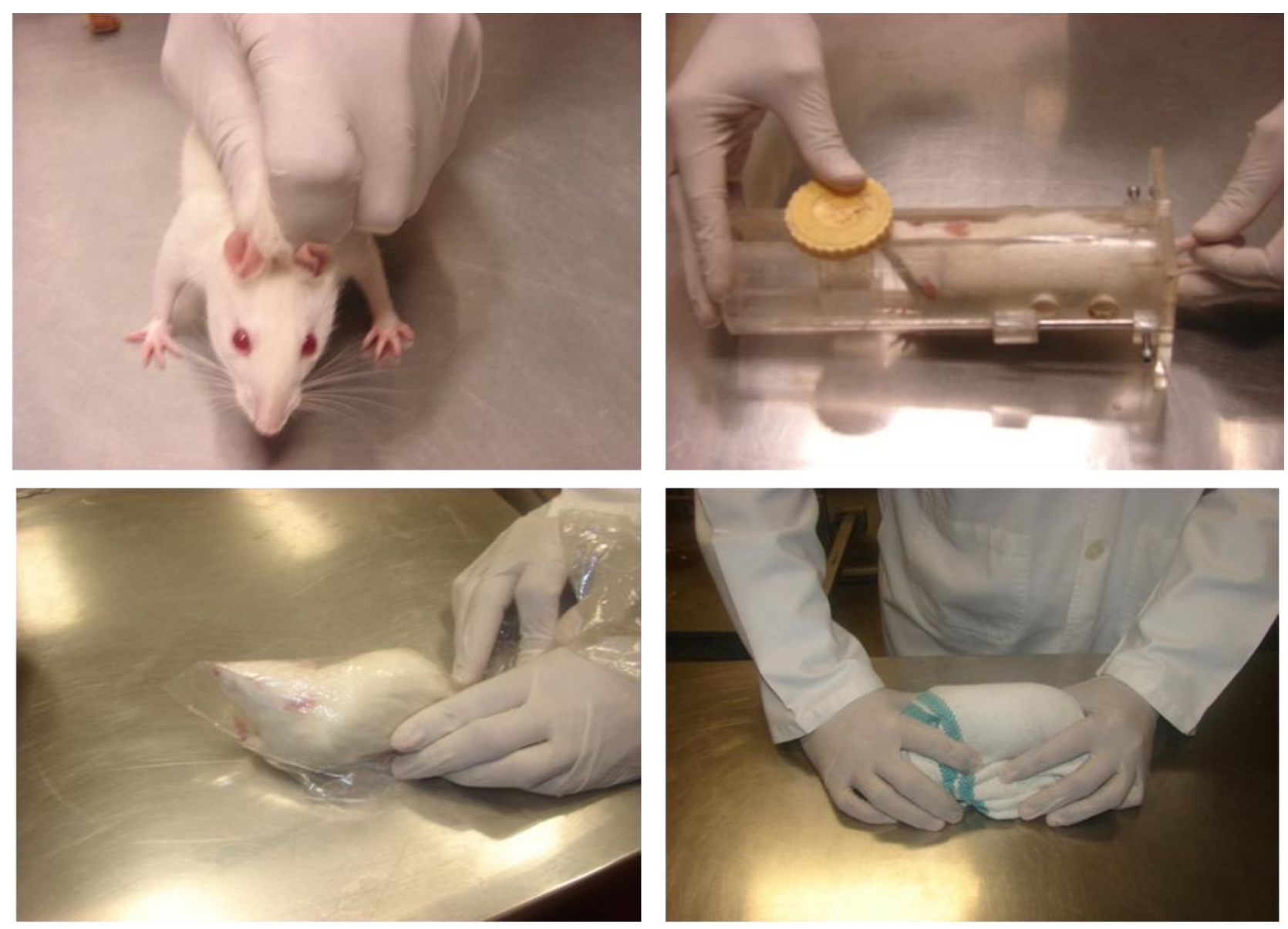

Fig.5: a: scruff restraint is considered to be a natural restraint technique, b: restraint box is used for blood collection or substance administration by tail's vein, c: cone restraint is suitable for small rats, $d$ : towels are also used for restraining highly aggressive and bad tempered rats. (Pictures generated at Aga Khan University, Karachi, Pakistan) 


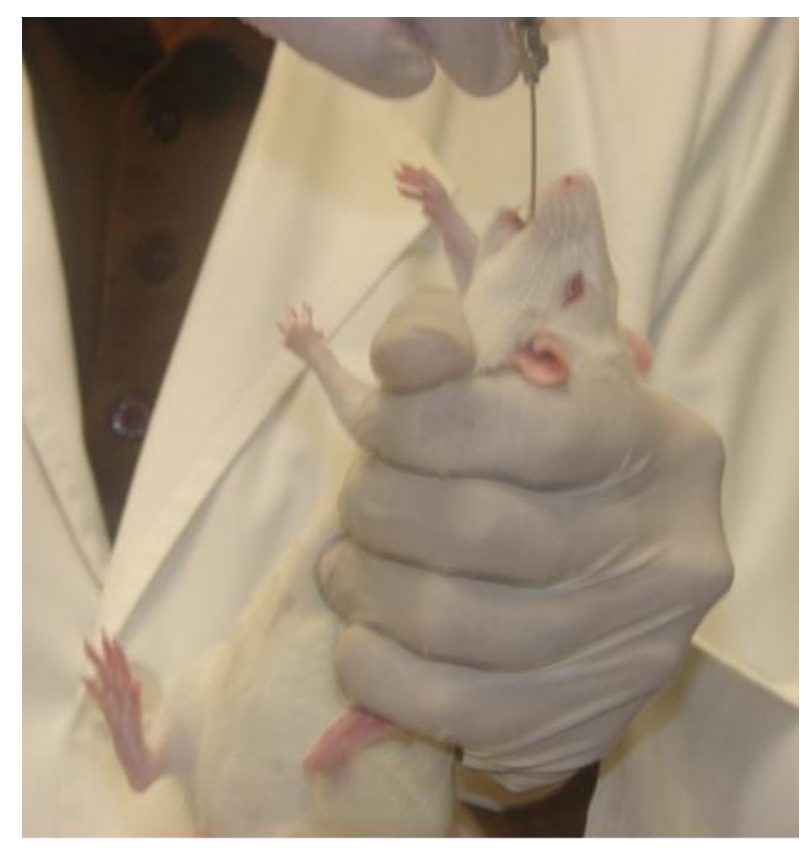

Fig.6: Substance administration: stainless steel made oral gavages are actively used for drug or diet administration. (Picture generated at Aga Khan University, Karachi, Pakistan) 

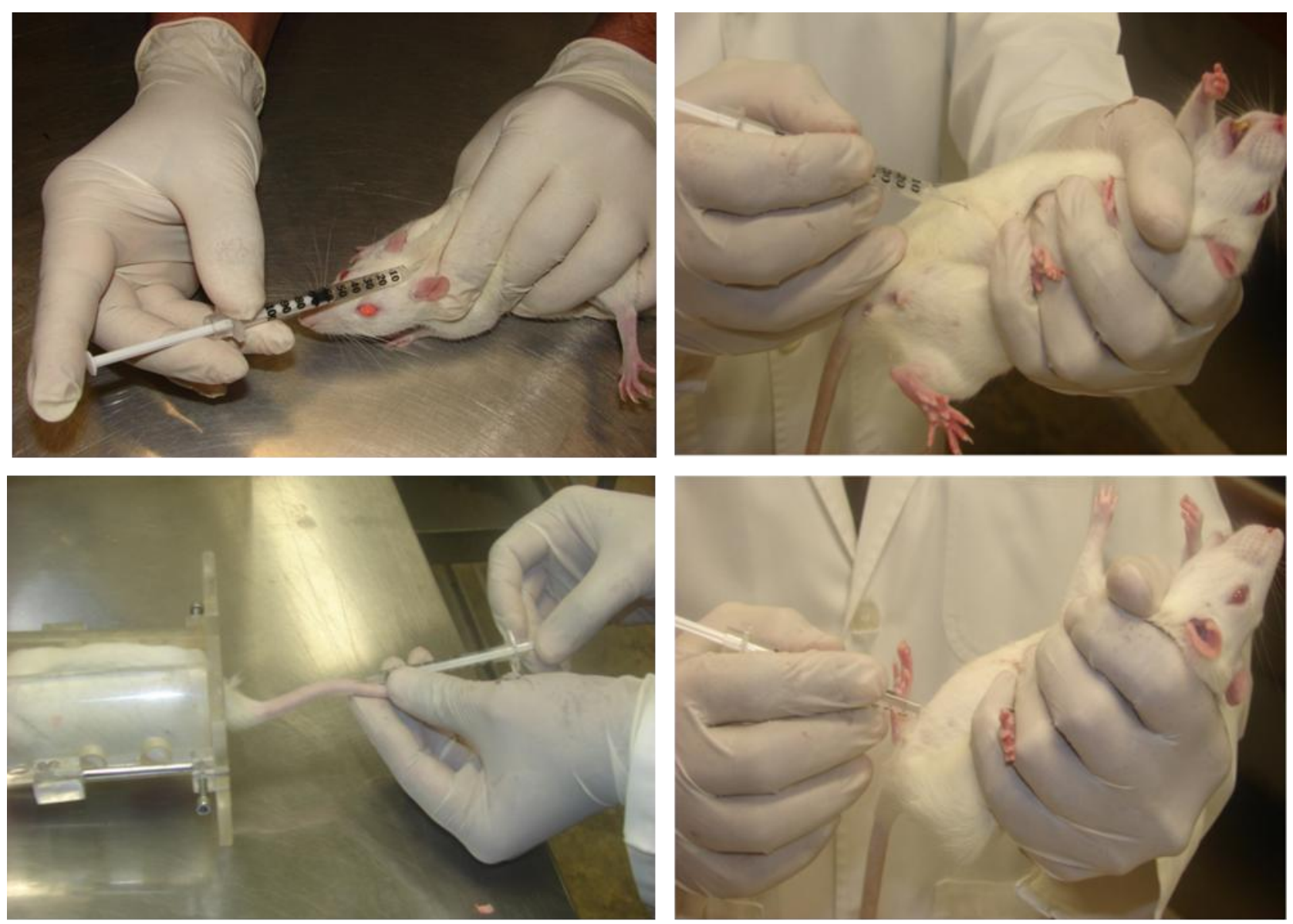

Fig.7: Substance administration: a: Low angle of the needle in tent of skin showing subcutaneous injection, b: Needle's insertion in the muscles of leg representing intramuscular injection, $c$ : Blood vein at Rat's tail is preferred site for intravenous injection, $d$ : intraperitoneal injection, the most commonly used drug administration method. (Pictures generated at Aga Khan University, Karachi, Pakistan) 